Article

\title{
Fabrication of Chitin/Poly(butylene succinate)/Chondroitin Sulfate Nanoparticles Ternary Composite Hydrogel Scaffold for Skin Tissue Engineering
}

\author{
S. Deepthi ${ }^{1}$, C. V. Sidhy Viha ${ }^{1}$, Chaochai Thitirat ${ }^{2}$, Tetsuya Furuike ${ }^{2}$, Hiroshi Tamura ${ }^{2, *}$ \\ and Rangasamy Jayakumar 1,2,*
}

1 Amrita Centre for Nanosciences and Molecular Medicine, Amrita Institute of Medical Sciences and Research Centre, Amrita Vishwa Vidyapeetham University, Kochi-682 041, India; E-Mails: deepthisankar@aims.amrita.edu (S.D.); sidhyvihacv@aims.amrita.edu (C.V.S.V.)

2 Faculty of Chemistry, Materials and Bioengineering, Kansai University, Osaka 564-8680, Japan; E-Mails: thitirat777@hotmail.com (C.T.); furuike@kansai-u.ac.jp (T.F.)

* Authors to whom correspondence should be addressed; E-Mails: tamura@kansai-u.ac.jp (H.T.); rjayakumar@aims.amrita.edu or jayakumar77@yahoo.com (R.J.); Tel.: +81-663-680-871 (H.T.); +91-484-280-1234 (ext. 8762) (R.J.); Fax: +81-663-303-770 (H.T.); +91-484-280-2020 (R.J).

External Editor: Kjell M. Vårum

Received: 4 November 2014; in revised form: 1 December 2014 / Accepted: 3 December 2014 / Published: 16 December 2014

\begin{abstract}
Skin loss is one of the oldest and still not totally resolved problems in the medical field. Since spontaneous healing of the dermal defects would not occur, the regeneration of full thickness of skin requires skin substitutes. Tissue engineering constructs would provide a three dimensional matrix for the reconstruction of skin tissue and the repair of damage. The aim of the present work is to develop a chitin based scaffold, by blending it with poly(butylene succinate) (PBS), an aliphatic, biodegradable and biocompatible synthetic polymer with excellent mechanical properties. The presence of chondroitin sulfate nanoparticles (CSnp) in the scaffold would favor cell adhesion. A chitin/PBS/CSnp composite hydrogel scaffold was developed and characterized by SEM (Scanning Electron Microscope), FTIR (Fourier Transform Infrared Spectroscopy), and swelling ratio of scaffolds were analyzed. The scaffolds were evaluated for the suitability for skin tissue engineering application by cytotoxicity, cell attachment, and cell proliferation studies using human dermal fibroblasts (HDF). The cytotoxicity and cell proliferation studies using HDF confirm the suitability of the scaffold for skin regeneration. In short, these results show
\end{abstract}


promising applicability of the developed chitin/PBS/CSnps ternary composite hydrogel scaffolds for skin tissue regeneration.

Keywords: chitin; poly(butylene succinate); skin tissue engineering; chondroitin sulfate; nanoparticle; composite

\section{Introduction}

As skin is the largest organ of the human body, skin regeneration holds a place of vital importance in tissue regeneration research. Skin tissue engineering projects the use of 3D scaffolds for the complete regeneration of affected sites. Ideal scaffolds should provide the required micro niche for the cells to proliferate and mature, with a constant supply of gas and nutrients. In this contest, the importance of a porous, 3D scaffold with native extracellular matrix (ECM) mimicking components come into the picture. Chitin, the second most abundant natural biopolymer after collagen, has been widely exploited due to its ECM-mimicking nature and one of the degradation products, viz, $\mathrm{N}$-acetyl-D-glucosamine, has been reported to promote fibroblast proliferation and ordered collagen deposition [1]. Chitin also promotes macrophage migration and stimulates the deposition of granulation tissue. Chitin also has shown a role in vascularization and has good haemostatic activity [2]. Optimal biomaterial design focuses on the use of bio-inert materials to minimize inflammatory response alleviated by foreign body reactions (FBR), thus, chitin, being bio-inert, and bio-active would be a suitable candidate. Chitin, due to its low amount of 2-amino-2-deoxy-D-glucose, is less soluble in acidic solvents, lacking the property of protonation [3]. The abundant hydroxyl group and $N$-acetyl group that are involved in inter and intra-molecular hydrogen bonding explains the high crystallinity of such aggregates. Chitin has been widely used in various tissue engineering and drug delivery applications, in the form of scaffolds [4-6], gels [7-9], fibers [10], etc. The high crystallinity of chitin compromises the mechanical integrity of such scaffolds. Aliphatic esters, a class of biodegradable synthetic polymers, have found profound applications, mainly due to their promising mechanical properties. The ester bonds in these synthetic polymers hydrolyze into non-toxic natural metabolites, which are either assimilated into the various respiration cycles in the human body and, finally, are eliminated from the body [11]. Poly(butylene succinate) (PBS) has been known for its processability, compared to poly(lactic acid) and poly(glycolic acid), and has been applied in tissue engineering scaffold fabrication in the form of fibers [12-14], scaffolds [15-17], and is often used as a blending material [18,19]. Succinic acid, the major degradation product, is an intermediate of tricarboxylic acid cycle or Krebs cycle and, thus, can be easily eliminated from body as carbon dioxide and water [20]. Various reports suggest the use of PBS to blend with natural polymers, such as chitosan, to impart strength to the composite $[13,15,18]$. These studies also reported that the implanted scaffolds displayed a normal to mild tissue response, with the development of chronic inflammatory response and FBR. In this study we have employed a simple technique to blend a natural polymer, chitin, with biodegradable aliphatic ester PBS by blending them in their dissolved states. Nanoparticles, by their large surface to volume ratio, extend different functional moieties on their surfaces for interaction. This would act as reinforcement for the composite prepared. To prove this hypothesis, we mixed chondroitin sulfate nanoparticles with the chitin/PBS 
composite. Chondroitin sulfate nanoparticles have been proven, in our previous report, to provide an enhanced fibroblast proliferation [1]. The developed ternary composite was then characterized and analyzed for cytocompatibility using Human Dermal Fibroblasts (HDF).

\section{Experimental Section}

\subsection{Materials}

$\alpha$-Chitin (85\% degree of acetylation) and chitosan (MW (molecular weight) 100-150 kDa, degree of deacetylation-85\%) were obtained from Koyo Chemical Co. Ltd., Koyo, Japan. Chondroitin sulfate was obtained from India Sea Food (Kochi, India). PBS (powder) was purchased from Sigma Aldrich (St. Louis, MO, USA). $\mathrm{CaCl}_{2}$, methanol and chloroform were purchased from Merck (Mumbai, India); glutaraldehyde from Fluka (St. Louis, MO, USA). DAPI (4',6-diamidino-2-phenylindole) was purchased from Gibco, Invitrogen (Bangalore, India). Human Dermal Fibroblasts and their media were purchased from Promo Cell (Heidelberg, Germany).

\subsection{Synthesis of Chitin/PBS/CSnp Ternary Composite Scaffold}

\subsubsection{Synthesis of Chitin/PBS Composite}

The synthesis of composite scaffold was done as reported earlier with modifications [4]. Briefly, chitin solution $(2 \% w / v)$ was prepared in methanol saturated with $\mathrm{CaCl}_{2}$. PBS was dissolved in chloroform $(0.5 \% w / v)$. Different ratios of chitin and PBS solutions were mixed to form gels of $10 \%-30 \% v / v$ of PBS. The solutions were blended under heating, and stirred continuously until a gel was formed; the latter was dialyzed against distilled water for 3 days. The hydrogel, thus obtained, was used for further experiments.

\subsubsection{Preparation of Chondroitin Sulfate Nanoparticle (CSnps)}

CSnps were prepared by ionic gelation method, as reported [1]. Briefly, the $\mathrm{pH}$ of $0.1 \%$ of chondroitin sulfate aqueous solution was reduced to 2 . Under continuous stirring, $0.05 \%$ chitosan in acetic acid solution was added until turbidity was observed. This would yield nanoparticles of size 100-150 nm with a polydispersity index of 0.28 .

\subsubsection{Preparation of Chitin/PBS/CSnps Ternary Composite}

The schematic representation of the procedure is shown in Figure 1. To the chitin/PBS hydrogel blend, CSnps suspension ( $10 \% v / w$ of hydrogel) was added and stirred overnight to obtain a homogenous mixture. The resulting slurry was then poured into a mold, frozen, and lyophilized for $24 \mathrm{~h}$.

\subsection{Swelling Studies}

The water uptake or swelling analysis was done in a neutral $\mathrm{pH}$ at $37^{\circ} \mathrm{C}$. Lyophilized sample was weighed $\left(W_{0}\right)$ and immersed in Phosphate Buffered Saline (PBS) for predetermined time intervals. 
The wet weight $\left(W_{1}\right)$ was noted after wiping off the excess PBS using a tissue paper. The swelling ratio was calculated using Equation (1).

$$
\text { Swelling ratio }=\left(W_{1}-W_{0}\right) / W_{0}
$$

Figure 1. Schematic representation of scaffold fabrication.
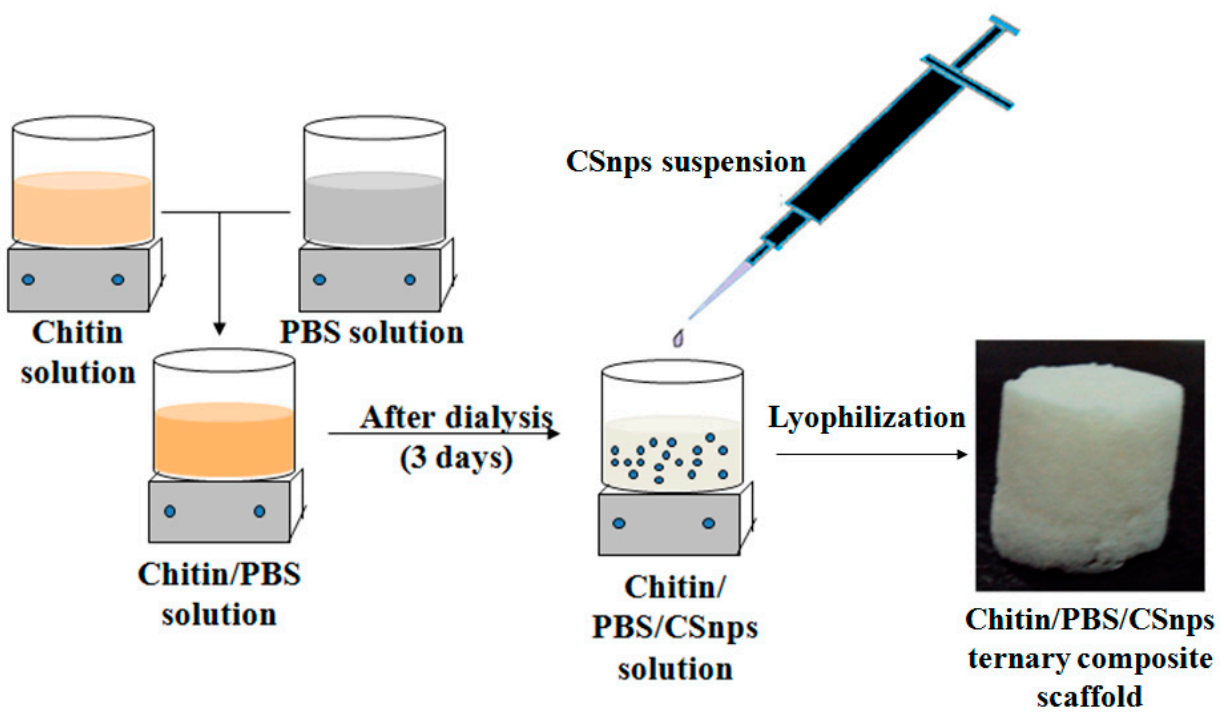

\subsection{Analytical Characterization}

The morphology of the developed scaffold was studied using a Scanning Electron Microscope (SEM) (JEOL, JSM-6490LA, Tokyo, Japan). A cross section of the lyophilized composite scaffold was taken from the interior and gold sputter coated (JEOL, JFC-1600) prior to imaging. FTIR measurements were performed using an FTIR spectrometer (Perkin-Elmer RX1, Waltham, MA, USA). The samples were finely ground and mixed with potassium bromide and pelletized. The pellets were scanned in the range of $400-4000 \mathrm{~cm}^{-1}$.

\subsection{Cell Viability Study}

Cell viability on chitin/PBS/CSnps scaffolds was evaluated using alamar blue assay. HDF cells were seeded at a density of 7500 cells/scaffold in a 96 well plate and incubated for predetermined time points. Viability was analyzed by replacing media after 24 and $48 \mathrm{~h}$ with $200 \mu \mathrm{L}$ fibroblast growth medium containing $10 \%$ alamar solution. After $6 \mathrm{~h}$ of incubation, the optical density was measured at $570 \mathrm{~nm}$ and reference at $600 \mathrm{~nm}$ in a microplate reader (Biotek PowerWave XS, Winooski, VT, USA). Cell viability was assessed from a histogram plotted as percentage viability with respect to control (cells in media) vs. incubation time.

\subsection{Live Dead Assay}

Live dead assay was performed on chitin/PBS scaffold with and without CSnps. HDFs were seeded onto the scaffolds at a seeding density of 15,000 cells per scaffold and, after the required incubation period (24 and $48 \mathrm{~h}$ ), they were given a PBS wash and stained with Calcein-AM and Ethidium homodimer at 
concentrations of $2 \mu \mathrm{M}$ and $4 \mu \mathrm{M}$, respectively, and incubated at $37{ }^{\circ} \mathrm{C}$ for $30 \mathrm{~min}$. The scaffolds were then washed and viewed under a fluorescent microscope (Olympus BX-51, Tokyo, Japan).

\subsection{Cell Attachment and Proliferation Study}

HDF cells were seeded at a density of 15,000 cells per scaffold in a 24 well plate and cultured for a predetermined time period $(24 \mathrm{~h})$. The scaffolds were then washed and fixed with $0.25 \%$ gluteraldehyde. The scaffolds were dehydrated and dried at room temperature. Cell attachment to scaffolds were visualized in SEM imaging. Fluorescence imaging was done after culturing cells on the scaffolds for 24 and $48 \mathrm{~h}$. After the predetermined time points, the scaffolds were washed with PBS twice and fixed with $4 \%$ paraformaldehyde. Cells were permeabilized using $0.5 \%$ Triton $\mathrm{X}$, the nucleus stained using DAPI, and viewed under fluorescent microscope (Olympus BX-51).

\subsection{Statistical Analysis}

Results are expressed as mean $\pm \mathrm{SD}$ of $n \geq 3$, where SD stands for standard deviation and $\mathrm{n}$ is the sample size. Statistical analysis was done using Student's paired $t$ test with a confidence level of 95\% and $99 \%$. The error bars denote \pm SD.

\section{Results and Discussion}

\subsection{Preparation and Characterization of Chitin/PBS/CSnps Ternary Composite}

The blending of chitin and PBS yielded a hard gel with reduced porosity as observed from the SEM image. The concentration of PBS has an indirect influence on the porosity of the scaffold (Figure 2). The higher concentration of PBS also showed precipitation of PBS on the surface of the scaffold, leading to irregularities in scaffold morphology. Of the different concentrations tried, the $20 \%$ PBS-containing scaffold was chosen for further studies. The incorporation of CSnps (size 100-150 nm) to the composite hydrogel increased the porosity after lyophilization, accounting for the water content replenished by adding CSnps suspension, and the pore size and porosity, as observed in the chitin hydrogel (Figure 3A), was regained in the ternary composite (Figure 3C). An optimum porosity is a prerequisite for better gas and nutrient exchange [4]. The macro-porous nature of the scaffold also aid in proper cell infiltration, and a homogeneous cell-laden construct could be achieved. Further the addition of nanoparticle increased the roughness of the scaffold (Figure 3D,E). This would increase the protein adsorption of the scaffold and, thereby, increase cell attachment.

Figure 2. SEM image of different concentration of PBS in chitin/PBS (A) $10 \%$, (B) 20\%, and (C) $30 \%$.
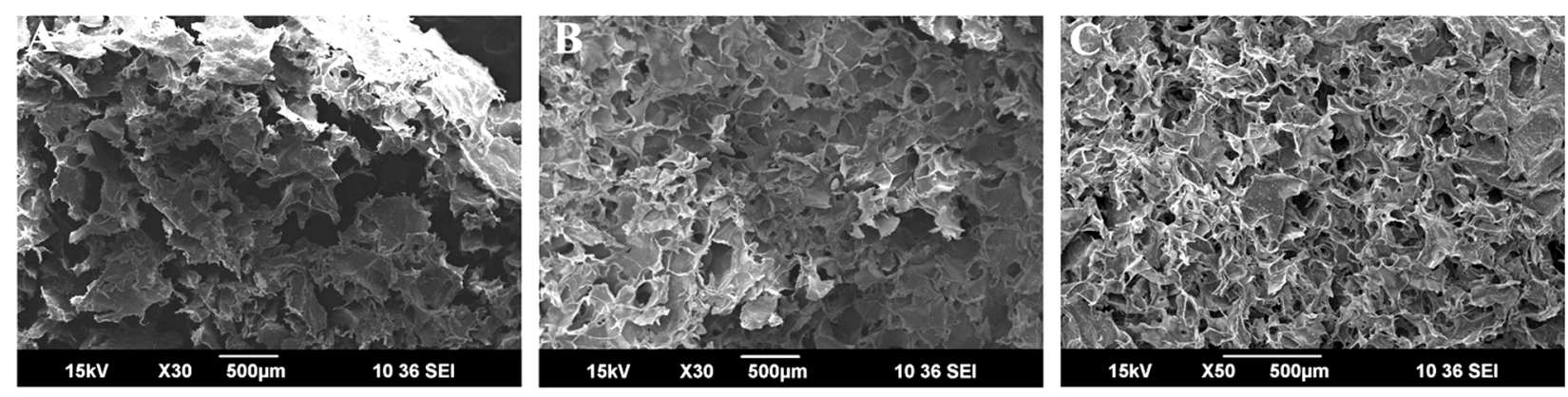
Figure 3. SEM image of (A) chitin, (B,D) chitin/PBS, and (C,E) Chitin/PBS/CSnps.
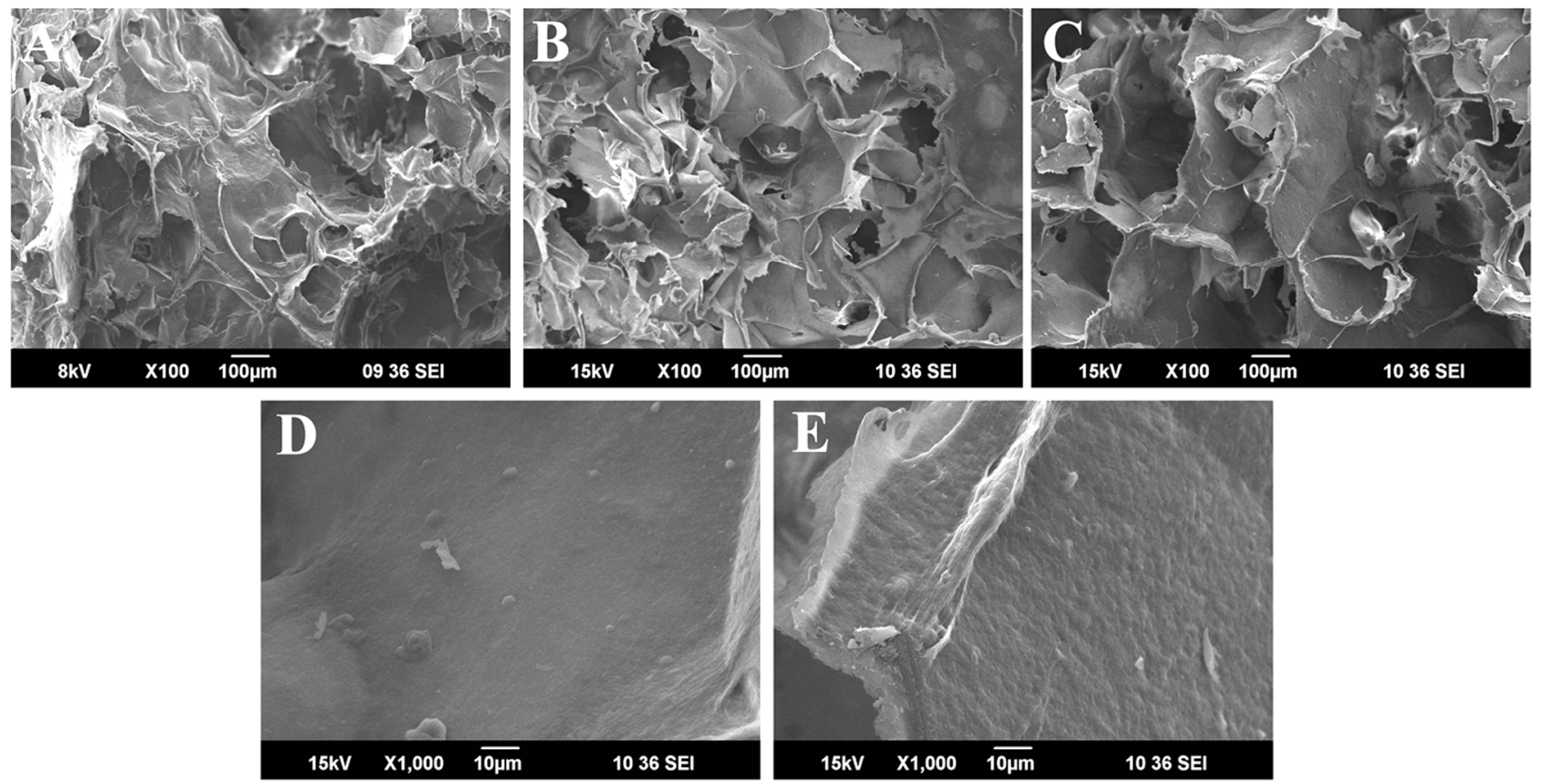

FTIR analysis of the composite showed the characteristic peaks of chitin and PBS (Figure 4). There was a shift in the $-\mathrm{C}=\mathrm{O}$ stretch (ester) of $\mathrm{PBS}$, from 1750 to $1725 \mathrm{~cm}^{-1}$, in the composite scaffold, demonstrating an interaction via hydrogen bonding. $-\mathrm{C}-\mathrm{O}$ ester stretches of PBS at 1326 and $1148 \mathrm{~cm}^{-1}$ were also observed in the composite scaffold [21]. All sharp intensity peaks observed in PBS were seen in the chitin/PBS composite, indicating that PBS was incorporated uniformly in the composite. The chitin specific bands were also observed in the FTIR of the composite. The bands include the amide I region, $1645 \mathrm{~cm}^{-1}$, and amide II region, $1565 \mathrm{~cm}^{-1}$. The sharp band at $1390 \mathrm{~cm}^{-1}$ depicts $-\mathrm{CH}_{3}$ symmetrical deformation. The existence of $\beta$ (1-4) glycosidic bond was confirmed by the band at $1069 \mathrm{~cm}^{-1}$. The broad peak of $-\mathrm{OH}$ stretching at $3400 \mathrm{~cm}^{-1}$ was also seen in the composite. The characteristic peak showing the $\mathrm{S}=\mathrm{O}$ stretching of sulfate group, indicating the presence of CSnps, was seen at $1246 \mathrm{~cm}^{-1}$ in the composite. All these confirm the uniform blending of the individual polymers in the ternary composite.

Figure 4. FTIR spectra of composite sponge (a) chitin, (b) PBS, (c) chitin/PBS, (d) chondroitin sulfate, and (e) chitin/PBS/CSnps.

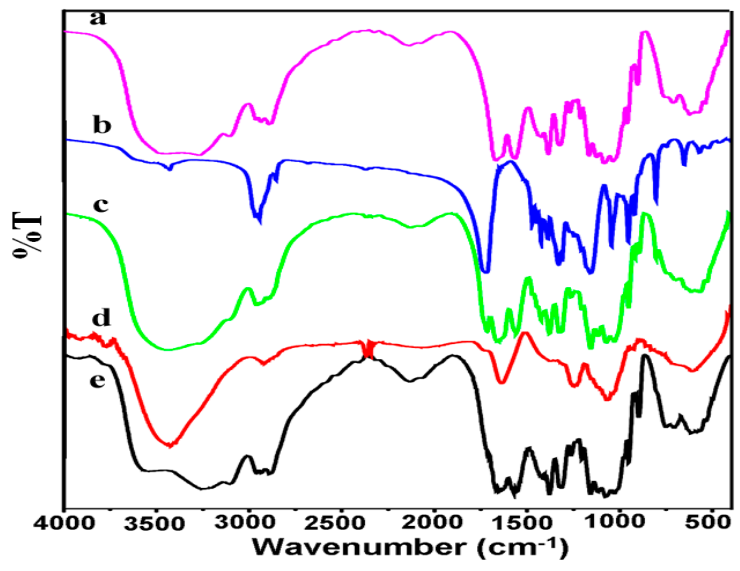


Swelling ratio remained more or less constant throughout the time period of observation, as observed in Figure 5A. Chitin showed the highest swelling ratio. The incorporation of PBS slightly reduced the swelling ability of the construct, but the addition of CSnps increased the swelling ability. By three weeks, the swelling ratio of chitin had started to reduce, indicating the onset of degradation. A controlled swelling is important for all tissue engineering constructs in order to absorb exudates, if any, and to provide a moist environment for better nutrient uptake and exchange [3].

Figure 5. (A) Swelling study of chitin, chitin PBS and chitin/PBS/CSnps in PBS and (B) Alamar blue assay to determine viability of HDFs on chitin, chitin PBS and chitin/PBS/CSnps after 24 and $48 \mathrm{~h}$. * indicates $p<0.05$ and $* *$ indicates $p<0.01$.
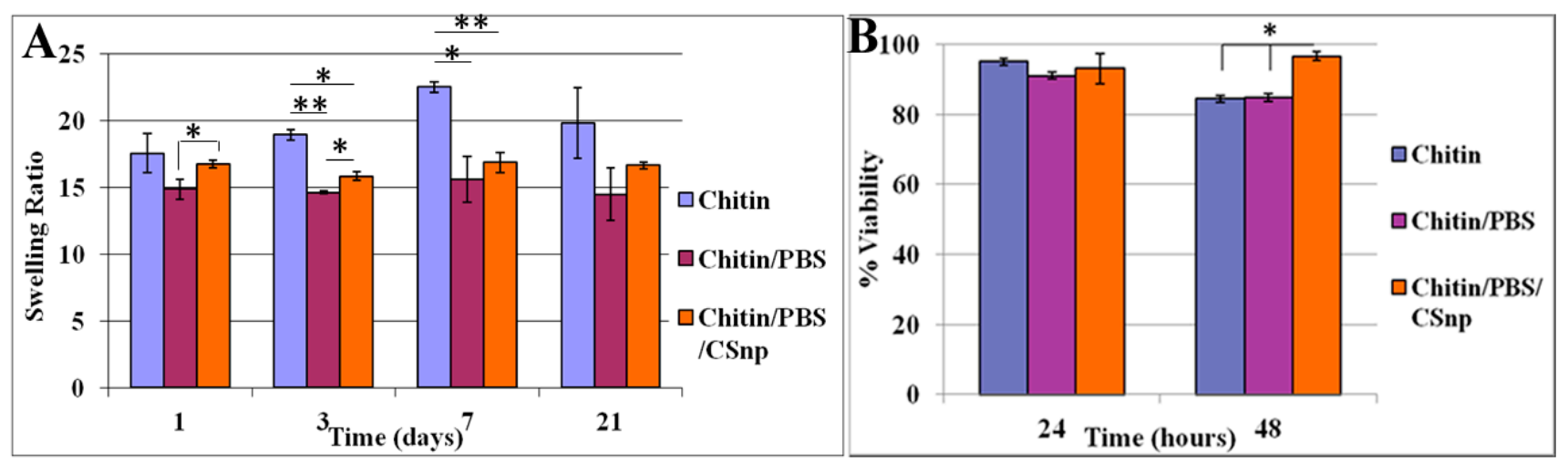

\subsection{Biocompatibility of the Composite}

The ternary composite showed a lesser reduction in percentage viability between 24 and $48 \mathrm{~h}$, compared to control chitin and chitin/PBS (Figure 5B). At $24 \mathrm{~h}$ there was no significant change in viability between the three samples. The $48 \mathrm{~h}$ sample containing CSnps showed the highest viability. This is also evident from the live dead assay, shown in Figure 6, wherein chitin and chitin/PBS scaffolds showed more dead cells (red). Chitin/PBS/CSnps ternary composite showed the highest live cell number (green) at both 24 and $48 \mathrm{~h}$. These results confirm the cytocompatible nature of the developed composite. The presence of CSnps in the composite in addition to increasing the porosity of the composite also increased the surface area for more cell adhesion. Fibroblasts retained the spindle shaped morphology in the ternary composite, demonstrating the cell-friendly nature of scaffold.

SEM image depicting the HDF attachment on the prepared scaffolds, HDF attachment on the chitin scaffold showed clumping or cell aggregations (Figure 7A,D). Compared to chitin, chitin/PBS scaffold showed better cell attachment (Figure 7B,E). More uniform spreading was evident in the blend. CSnps in the ternary composite scaffold enhanced cell attachment by changing the roughness of the composite. The cells tend to attach very well and spread in the ternary composite (Figure 7C,F). The higher magnification clearly depicts the uniform attachment of HDFs on the ternary composite with strong filopodial extensions (Figure 7F, arrow). The fluorescent image shows the extent of HDF proliferation (Figure 8). The chitin scaffold showed an aggregation of cells at the initial time point (Figure 8A). Blending of PBS in the chitin showed a remarkable difference in the cell attachment. The initial cell attachment had drastically increased, though the reduced porosity hindered further cell proliferation, reducing the cell number in $48 \mathrm{~h}$ (Figure 8B,E). CSnps incorporated composite showed improved cell 
attachment and a homogeneous distribution of cells was observed within $48 \mathrm{~h}$ (Figure 8F). Chondroitin sulfate being an ECM component, has been known to accelerate wound healing by enhancing the influx of fibroblasts into the wound, increase synthesis of proteoglycan, collagen-II and also exert anti-inflammatory activity [1]. The development of chondroitin sulfate in the nano-regime would exemplify the properties of the same and further increase the surface area for interaction. Thus, the scenario observed here by the incorporation of CSnps in the chitin/PBS scaffold would be the additive effect of the properties of chondroitin sulfate and chitin in fibroblast proliferation, thereby promoting skin regeneration.

Figure 6. Live dead assay of HDFs on (A,D), Chitin (B,E), Chitin/PBS (C,F), Chitin/PBS/CSnps after $24 \mathrm{~h}(\mathbf{A}-\mathbf{C})$, and $48 \mathrm{~h}$ (D-F). Scale bar denotes $50 \mu \mathrm{m}$.
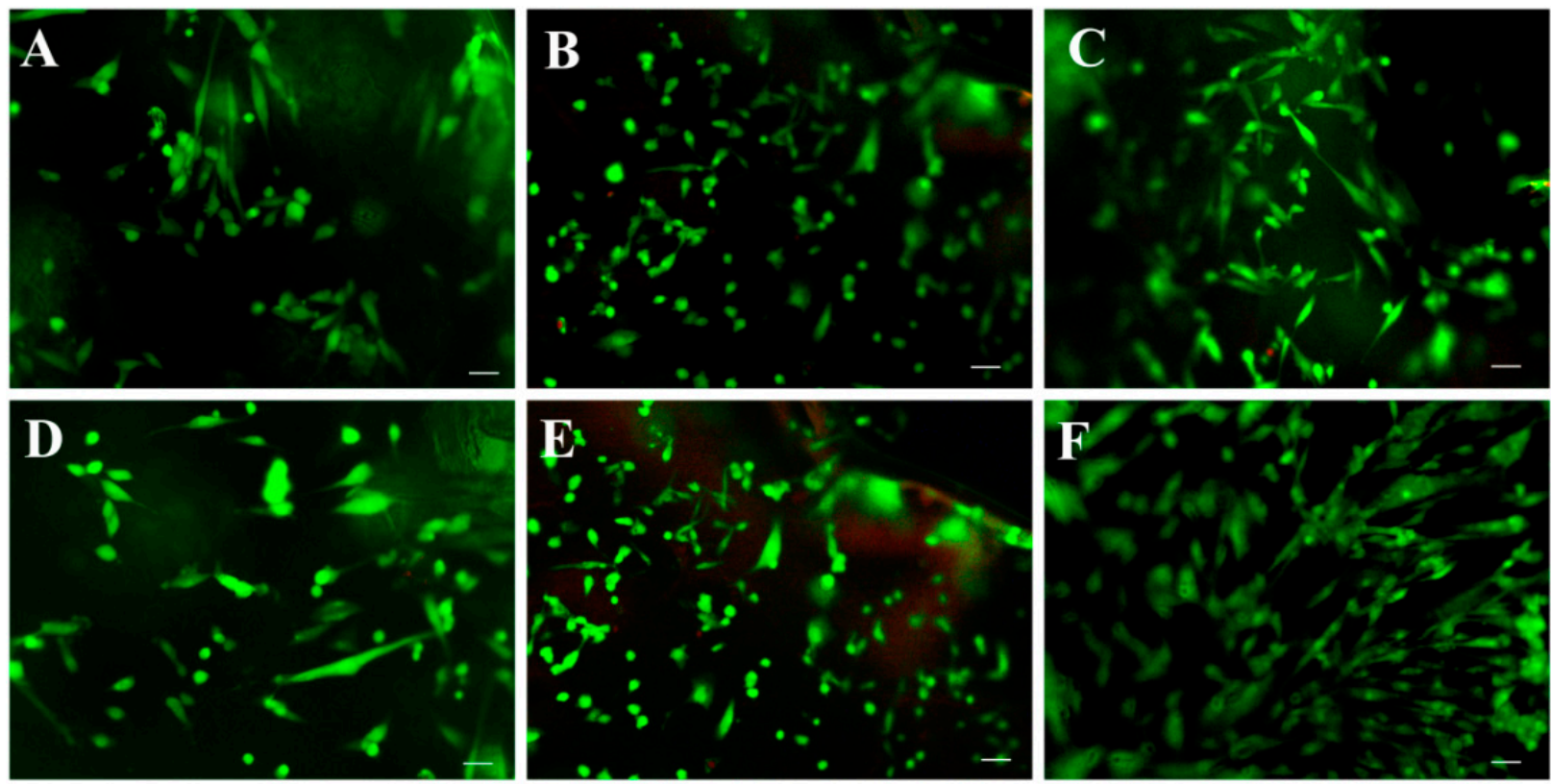

Figure 7. SEM image showing the HDF attachment on the composite scaffold at $24 \mathrm{~h}$. (A,D) Chitin, (B,E) Chitin/PBS, and (C,F) Chitin/PBS/CSnp.
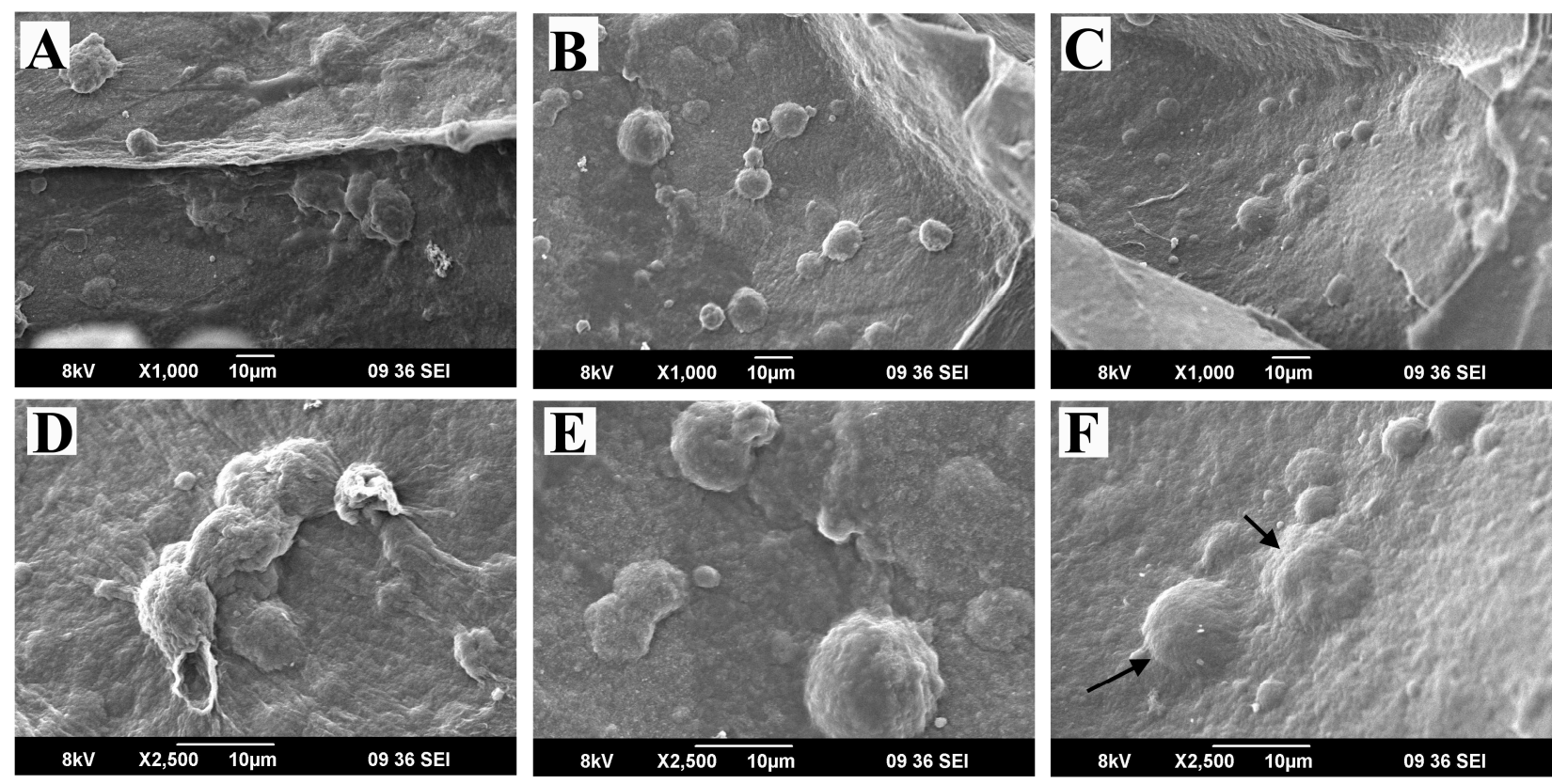
Figure 8. DAPI nucleus staining showing the attachment of HDF after $24 \mathrm{~h}(\mathbf{A}-\mathbf{C})$ and $48 \mathrm{~h}(\mathbf{D}-\mathbf{F})$ on $(\mathbf{A}, \mathbf{D})$ Chitin, (B,E) Chitin/PBS, and (C,F) Chitin/PBS/CSnps. Scale bar denotes $50 \mu \mathrm{m}$.
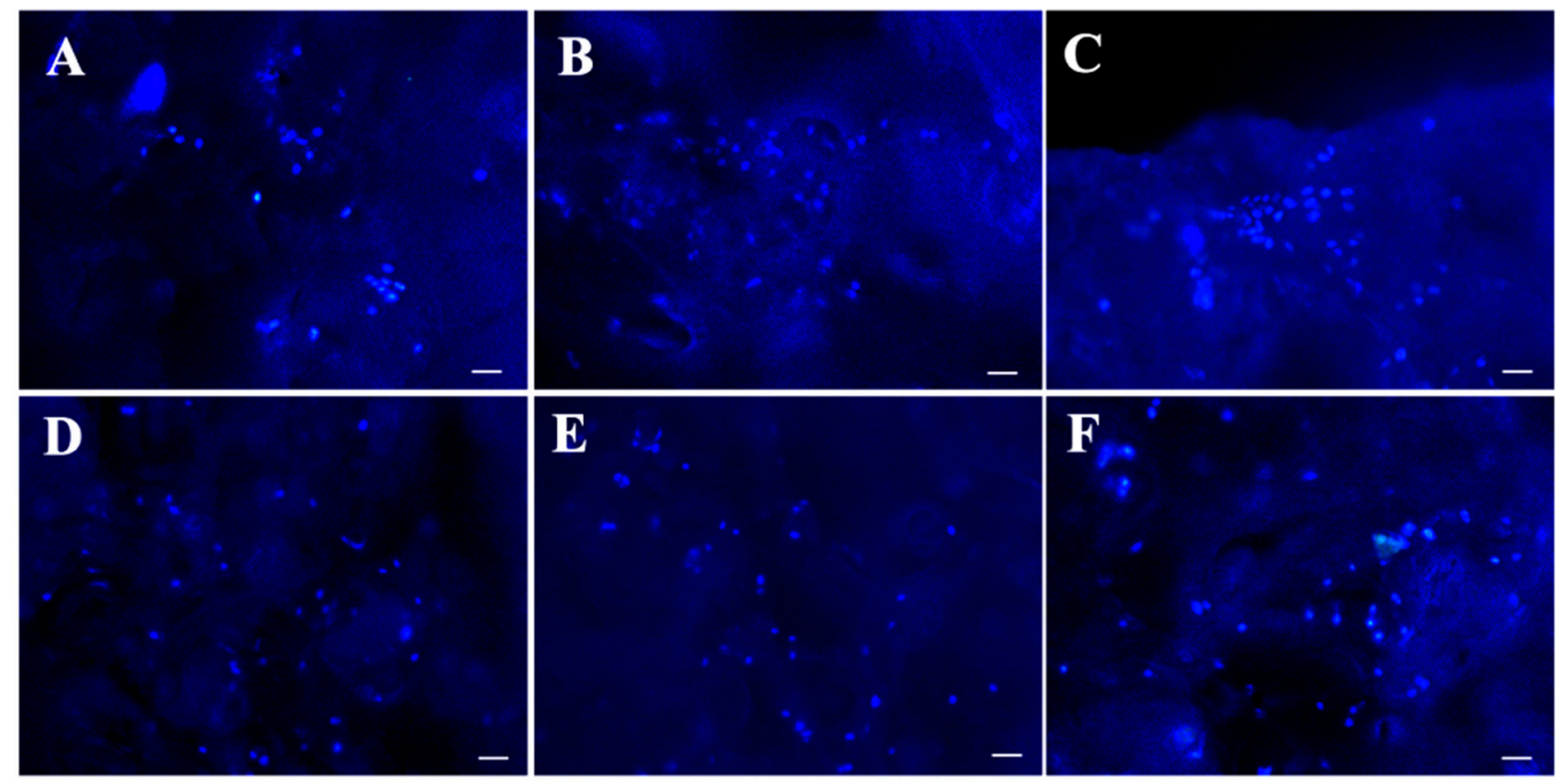

\section{Conclusions}

A uniform blend of chitin and PBS was achieved and a ternary composite containing CSnps was developed and characterized. FTIR results confirmed the uniform blending of chitin and PBS and the base of interaction was observed to be hydrogen bonding. The incorporation of CSnps increased the porosity of the blend scaffold. Optimum porosity and controlled swelling was achieved, which would help in the proper gas and nutrient exchange. The macro-porous and bioactive nature of the ternary composite also increased HDF attachment and proliferation, thereby, making it a homogeneous cell-laden construct. This work reports a simple technique to develop a macro-porous ternary composite scaffold for skin tissue engineering.

\section{Acknowledgments}

The author R. Jayakumar is grateful to Kansai University, Osaka, for supporting this project under the "Visiting Researcher Program". One of the authors, Deepthi Sankar, is also grateful to the Council of Scientific and Industrial Research for supporting, financially, under the CSIR-SRF Award No. 9/963(0034)2K13-EMR-I.

\section{Author Contributions}

All measurements and characterizations were carried out by S. Deepthi, C.V. Sidhy Viha, and Chaochai Thitirat. Furthermore, they contributed to the writing of the paper. Tetsuya Furuike, Hiroshi Tamura, and R. Jayakumar coordinated the study and the writing of the manuscript. 


\section{Conflicts of Interest}

The authors declare no conflicts of interest.

\section{References}

1. Anisha, B.S.; Deepthi, S.; Annapoorna, M.; Chennazhi, K.P.; Nair, S.V.; Jayakumar, R. Chitosan-hyaluronic acid/nano chondroitin sulphate ternary composite sponges for biomedical use. Carbohydr. Polym. 2013, 92, 1470-1476.

2. Yang, T.L. Chitin-based Materials in tissue engineering: Applications in soft tissue and epithelial organ. Int. J. Mol. Sci. 2011, 12, 1936-1963.

3. Anitha, A.; Sowmya, S.; Sudheeshkumar, P.T.; Deepthi, S.; Chennazhi, K.P.; Ehrlich, H.; Tsurkan, M.; Jayakumar, R. Chitin and chitosan in selected biomedical applications. Prog. Polym. Sci. 2014, 39, 1644-1667.

4. Deepthi, S.; Chennazhi, K.P.; Nair, S.V.; Jayakumar, R. Fabrication of chitin/poly(3-hydroxybutyrateco-3-hydroxyvalerate) hydrogel scaffold. Carbohydr. Polym. 2012, 90, 725-729.

5. Jayakumar, R.; Menon, D.; Manzoor, K.; Nair, S.V.; Tamura, H. Biomedical applications of chitin and chitosan based nanomaterials-A short review. Carbohydr. Polym. 2010, 82, 227-232.

6. Madhumathi, K.; Sudheesh Kumar, P.T.; Abhilash, S.; Sreeja, V.; Tamura, H.; Manzoor, K.; Nair, S.V.; Jayakumar, R. Development of novel chitin/nanosilver composite scaffolds for wound dressing applications. J. Mater. Sci. Mater. Med. 2010, 21, 807-813.

7. Jayakumar, R.; Amrita, N.; Sanoj Rejinold, N.; Maya, S.; Nair, S.V. Doxorubicin-loaded pH-responsive chitin nanogels for drug delivery to cancer cells. Carbohydr. Polym. 2012, 87, 2352-2356.

8. Tamura, H.; Furuike, T.; Nair, S.V.; Jayakumar, R. Biomedical applications of chitin hydrogel membranes and scaffolds. Carbohydr. Polym. 2011, 84, 820-824.

9. Arunraj, T.R.; Sanoj Rejinold, N.; Ashwin Kumar, N.; Jayakumar, R. Bio-responsive chitin-poly (L-lactic acid) composite nanogels for liver cancer. Colloids Surf. B Biointerf. 2014, 113, 394-402.

10. Shalumon, K.T.; Binulal, N.S.; Selvamurugan, N.; Nair, S.V.; Menon, D.; Furuike, T.; Tamura, H.; Jayakumar, R. Electrospinning of carboxymethyl chitin/poly (vinyl alcohol) nanofibrous scaffolds for tissue engineering applications. Carbohydr. Polym. 2009, 77, 863-869.

11. Correlo, V.M.; Boesel, L.F.; Bhattacharya, M.; Mano, J.F.; Neves, N.M.; Reis, R.L. Properties of melt processed chitosan and aliphatic polyester blends. Mater. Sci. Eng. A 2005, 403, 57-68.

12. Tian, L.; Wang, P.; Zhao, Z.; Ji, J. Antimicrobial activity of electrospun poly(butylenes succinate) fiber mats containing PVP-capped silver nanoparticles. Appl. Biochem. Biotechnol. 2013, 171, 1890-1899.

13. Costa-Pinto, A.R.; Martins, A.M.; Castelhano-Carlos, M.J.; Correlo, V.M.; Sol, P.C.; Longatto-Filho, A.; Battacharya, M.; Reis, R.L.; Neves, N.M. In vitro degradation and in vivo biocompatibility of chitosan-poly(butylene succinate) fiber mesh scaffolds. J. Bioact. Compat. Polym. 2014, 29, 137-151. 
14. Jeong, E.H.; Im, S.S.; Youk, J.H. Electrospinning and structural characterization of ultrafine poly(butylene succinate) fibers. Polymer 2005, 46, 9538-9543.

15. Oliveira, J.T.; Correlo, V.M.; Sol, P.C.; Costa-Pinto, A.R.; Malafaya, P.B.; Salgado, A.J.; Bhattacharya, M.; Charbord, P.; Neves, N.M.; Reis, R.L. Assessment of the suitability of chitosan/poly(butylene succinate) scaffolds seeded with mouse mesenchymal progenitor cells for a cartilage tissue engineering approach. Tissue Eng. A 2008, 14, 1651-1661.

16. Xu, J.; Guo, B.H. Poly(butylene succinate) and its copolymers: Research, development and industrialization. Biotechnol. J. 2010, 5, 1149-1163.

17. Ikada, Y.; Tsuji, H. Biodegradable polyesters for medical and ecological applications. Macromol. Rapid Commun. 2000, 21, 117-132.

18. Coutinho, D.F.; Pashkuleva, I.H.; Alves, C.M.; Marques, A.P.; Neves, N.M.; Reis, R.L. The effect of chitosan on the in vitro biological performance of chitosan-poly(butylene succinate) blends. Biomacromolecules 2008, 9, 1139-1145.

19. Wang, H.; Gan, Z.; Schultz, J.M.; Yan, S. A morphological study of poly(butylene succinate)/ poly(butylene adipate) blends with different blend ratios and crystallization processes. Polymer 2008, 49, 2342-2353.

20. Costa-Pinto, A.R.; Correlo, V.M.; Sol, P.C.; Bhattacharya, M.; Srouji, S.; Livne, E.; Reis, R.L.; Neves, N.M. Chitosan-poly(butylene succinate) scaffolds and human bone marrow stromal cells induce bone repair in a mouse calvaria model. J. Tissue Eng. Regen. Med. 2012, 6, 21-28.

21. Zhao, P.; Liu, W.; Wu, Q.; Ren, J. Preparation, mechanical, and thermal properties of biodegradable polyesters/poly(lactic acid) blends. J. Nanomater. 2010, 2010, 1-8.

(C) 2014 by the authors; licensee MDPI, Basel, Switzerland. This article is an open access article distributed under the terms and conditions of the Creative Commons Attribution license (http://creativecommons.org/licenses/by/4.0/). 